\title{
Secreted factors from ventral telencephalon induce the differentiation of GABAergic neurons in cortical cultures
}

\author{
H.-h. Trinh, ${ }^{1, \star}$ J. Reid, ${ }^{2 \star}$ E. Shin, ${ }^{2}$ A. Liapi ${ }^{1}$ J. G. Parnavelas ${ }^{1}$ and B. Nadarajah ${ }^{2}$ \\ ${ }_{1}^{1}$ Department of Anatomy and Developmental Biology, University College London, Gower Street, London WC1E 6BT, UK \\ ${ }^{2}$ Faculty of Life Sciences, 1.124 Stopford Building, University of Manchester, Manchester M13 9PT, UK
}

Keywords: development, differentiation, interneurons, rat, telencephalon

\begin{abstract}
It is widely believed that the pyramidal cells and interneurons of the cerebral cortex are distinct in their origin, lineage and genetic make up. In view of these findings, the current thesis is that the phenotype determination of cortical neurons is primarily directed by genetic mechanisms. Using in vitro assays, the present study demonstrates that secreted factors from ganglionic eminence (GE) of the ventral telencephalon have the potency to induce the differentiation of a subset of cortical neurons towards $\gamma$-aminobutyric acid (GABA)ergic lineage. Characterization of cortical cultures that were exposed to medium derived from GE illustrated a significant increase in the number of GABA-, calretinin- and calbindin-positive neurons. Calcium imaging together with pharmacological studies showed that the application of exogenous medium significantly elevated the intracellular calcium transients in cortical neurons through the activation of ionotropic glutamate receptors. The increase in GABA+ neurons appeared to be associated with the elevated calcium activity; treatment with blockers specific for glutamate receptors abolished both the synchronized transients and reduced the differentiation of GABAergic neurons. Such studies demonstrate that although intrinsic mechanisms determine the fate of cortical interneurons, extrinsic factors have the potency to influence their neurochemical differentiation and contribute towards their molecular diversity.
\end{abstract}

\section{Introduction}

The adult cerebral cortex contains multiple classes of $\gamma$-aminobutyric acid (GABA)-containing interneurons that are distinct in morphology, physiology and cellular characteristics. On the basis of their chemical content, cortical interneurons are categorized into populations that are positive for parvalbumin, calbindin/somatostatin and calretinin/vasoactive intestinal peptide (DeFelipe, 1997). Further, it appears that interneurons that are diverse in their chemical content also differ in their electrophysiological properties (Kawaguchi \& Kondo, 2002; Markram et al., 2004). Evidence in recent years has shown that the pyramidal cells and the interneurons arise in discrete regions of the telencephalon (Flames \& Marin, 2005). GABAergic interneurons that populate the cortex arise from multiple regions of the ventral telencephalon, including the lateral, medial and caudal ganglionic eminences (GE). These cells migrate tangentially to populate the dorsal telencephalic structures including cerebral cortex. In view of these findings, it is likely that genetic mechanisms that are restricted to the progenitors of ventral telencephalon direct the specification of cortical interneurons.

It is evident that transcription factors $D l x 1 / 2$ and Mash1, expressed in the ventral telencephalon, direct the generation of interneurons, while a number of early genes are involved in the developmental regulation of these cells (Anderson et al., 1997; Fode et al., 2000; Schuurmans \& Guillemot, 2002). In accordance, in vivo genetic

Correspondence: Dr B. Nadarajah, as above.

E-mail: bagirathy.nadarajah@manchester.ac.uk

${ }^{*}$ H.-h.T. and J.R. contributed equally to this work.

Received 11 June 2006, revised 30 August 2006, accepted 25 September 2006 programmes that are specific to the dorsal telencephalon suppress the neurochemical differentiation of cortical neurons towards GABAergic lineage. In contrast to the prevailing view, previous cell culture data have illustrated that cortical progenitors do have the potency to generate both glutamatergic and GABAergic neural populations in proportions that are similar to those in vivo (Gotz et al., 1995; de Lima \& Voigt, 1997; Gulacsi \& Lillien, 2003). Interestingly, the production of a significant number of small GABA+ neurons in mature cortical cultures (de Lima \& Voigt, 1997) is consistent with a recent in vivo study that showed the generation of small GABA+ neurons in postnatal cerebral cortex (Dayer et al., 2005). Based on the premise that $D l x$ is required for the generation of GABA+ neurons, the latter study posits that GE-derived precursors in the cortex may retain the ability to generate new interneurons during postnatal life.

While extensive research has been carried out in the identification of genetic mechanisms that underlie the generation, specification and migration of cortical interneurons (Marin \& Rubenstein, 2001; Schuurmans \& Guillemot, 2002), the contribution of epigenetic factors in the specification of cortical interneurons remains less well known. Because the phenotypes of cortical interneurons are specified by genetic mechanisms that are restricted to the GEs of ventral telencephalon, we reasoned that epigenetic factors from ventral telencephalon might enable the differentiation of cortical GABAergic neurons. In this context, the previous work of Gulacsi \& Lillien (2003) has demonstrated that secreted sonic hedgehog from ventral telencephalon induces the differentiation of GABAergic neurons. Thus, to determine the influence of epigenetic factors in the differentiation of cortical GABAergic neurons, rat E16 cortical cells were exposed to conditioned medium derived from GE cultures. 
Treated cultures were subsequently analysed for GABA immunoreactivity and further assayed for cell proliferation, neuronal output and subtypes of cortical interneurons. To examine the underlying cellular mechanisms and receptor systems that might mediate the epigenetic influence, pharmacological experiments were carried out together with calcium-imaging studies.

\section{Materials and methods \\ Preparation of cortical cell cultures}

Time-mated Sprague-Dawley pregnant rats (day of the vaginal plug $=\mathrm{E} 1)$ were killed at gestation day 16 (E16) according to the scheduled guidelines of Home office, UK ( $\mathrm{CO}_{2}$ inhalation), and the embryos harvested in rat artificial cerebrospinal fluid. Cortices were removed and dissociated by enzymatic treatment with a medium containing DMEM/F12 (Sigma, UK), 0.05\% trypsin (ICN, UK) and $0.001 \%$ DNase I (Invitrogen, UK), for $1 \mathrm{~h}$. Trypsin was inactivated with $10 \%$ heat-inactivated foetal calf serum (Gibco, UK), and the tissue pieces were mechanically dissociated. The resulting cell suspension was centrifuged, resuspended and plated on glass coverslips coated with poly-L-lysine $(10 \mathrm{mg} / \mathrm{mL}$; Sigma) and laminin (5 mg/mL; Sigma) at a density of $1.5 \times 10^{5}$ cells. Cells were initially maintained in DMEM/F12, $10 \%$ foetal calf serum, $2 \mathrm{mM}$ L-glutamine (ICN) and penicillin/streptomycin (ICN). Cultures were then transferred to medium containing $1 \%$ serum for a further $24 \mathrm{~h}$, and thereafter maintained in serum-free medium supplemented with $\mathrm{N}-2$ (Gibco) until further treatment. E12-14 rat cortical cultures and postnatal (P)2-4 subventricular zone (PSVZ) cultures were prepared as described above; the PSVZ was excised from the anterior dorsal horn of the forebrain, and the progenitor content of the cultures was ascertained by cell proliferation assay. However, to increase the survival and to maintain the progenitor population, cultures were initially expanded in $20 \mathrm{ng}$ of epidermal growth factor before the application of GE conditioned medium. For control experiments, rat E16 spinal cord and mid-brain cultures were prepared as described above for embryonic cortical cultures.

\section{Preparation of GE cultures and collection of conditioned medium}

E16 GE cultures, prepared as described above, were plated on poly-Llysine- and laminin-coated plastic dishes and grown in a medium with reducing content of serum until the cultures were confluent. Thereafter, cells were maintained in serum-free medium supplemented with $\mathrm{N}-2$. Conditioned medium (termed as GE medium) was collected every other day for a duration of 11 days, pooled, aliquoted and stored at $-20{ }^{\circ} \mathrm{C}$ until further use.

\section{Experimental treatment}

To test the effects of secreted factors from ventral telencephalon, serum-free cortical cultures were treated with GE medium for five consecutive days; serum-free cultures maintained for the same length of period were used as controls.

\section{Cell proliferation analysis}

To assay cell proliferation, serum-free cultures were pulsed with bromodeoxyuridine (BrdU; $10 \mu \mathrm{M}$, Sigma) for $1 \mathrm{~h}$ before commen- cing the experimental treatment to label a cohort of progenitors progressing through the S-phase of cell cycle.

\section{Pharmacological studies - acute assessment of $\mathrm{Ca}^{2+}$ activity}

Serum-free cortical cultures and those grown in GE medium were exposed to the following drugs, respectively: tetrodotoxin (TTX; $1 \mu \mathrm{m}$, Sigma), bicuculline methiodide (BMI; $50 \mu \mathrm{m}$, Sigma), 2-amino-5-phosphonovalerate (AP5; $30 \mu \mathrm{m}$, Sigma), 1,2,3,4-tetrahydro-6-nitro-2,3-dioxo-benzo[f]quinoxaline-7-sulphonamide (NBQX; $50 \mu \mathrm{m}$; Sigma), K252a (200 nM, Tocris, UK), cadmium chloride (100 $\mu \mathrm{m}$, Sigma), CGP55845A (50 $\mu \mathrm{m}$, Tocris) and nipecotic acid (50 $\mu \mathrm{m}$, Sigma). Cells were perfused with drugs for $15 \mathrm{~min}$ and then returned to control or GE medium until partial recovery of $\mathrm{Ca}^{2+}$ activity was achieved.

\section{Pharmacological studies - chronic treatment}

Serum-free cortical cultures were maintained in the following antagonists for 5 days alone (controls) or in concert with GE medium (treatment group): BMI $(50 \mu \mathrm{m})$, AP5 $(30 \mu \mathrm{m}), \mathrm{NBQX}(50 \mu \mathrm{m})$, K252a $(200 \mathrm{nM})$, CGP55845A $(50 \mu \mathrm{m})$ or nipecotic acid $(50 \mu \mathrm{m})$. At the end of the treatment cultures were imaged for calcium activity or fixed and processed for immunohistochemistry.

\section{Calcium imaging}

Cortical cells grown in control or treatment medium were labelled with Oregon Green BAPTA $(10 \mu \mathrm{M} / \mathrm{mL}$; Molecular Probes, UK) for $20 \mathrm{~min}$. Labelled coverslips were transferred to a temperaturecontrolled glass chamber $\left(35-37{ }^{\circ} \mathrm{C}\right)$ and imaged using a Nikon EC600 microscope $(\times 40$ water objective) equipped with cooled CCD system. Images were collected every second for $5 \mathrm{~min}$ to assess baseline activity before the acute application of drugs. Data were analysed using Metamorph imaging software (Universal Imaging Corporation, USA), and are presented as percentage change in fluorescence intensity over baseline fluorescence $(\Delta F / F \%)$.

\section{Immunocytochemistry}

Cultured cells were fixed with $4 \%$ paraformaldehyde in $0.1 \mathrm{M}$ phosphate buffer ( $\mathrm{pH}$ 7.4), and processed for immunohistochemistry using standard protocol. Cells were incubated overnight with primary antibodies against GABA (rabbit polyclonal, 1:1000, Sigma), calretinin, calbindin (rabbit polyclonal, $1: 1000$, Swant, Bellinzona, Switzerland), $\mathrm{GAD}_{65 / 67}$ (rabbit polyclonal, $1: 500$, Chemicon, UK), Neu-N (mouse monoclonal, $1: 400$, Chemicon) or BrdU (mouse monoclonal, $1: 300$, Progen Biotechnik GMBH, Germany). After multiple washes, cells were incubated with FITC or rhodamineconjugated ALEXA secondary antibodies (1 : 500, Molecular Probes) for $2 \mathrm{~h}$ at room temperature. Labelled cells were examined with a Nikon EC600 fluorescent microscope equipped with cooled CCD system. Images were subsequently analysed using Metamorph imaging software.

\section{Quantitative analysis}

To examine the effects of GE medium on E16 cortical cultures, a minimum of 10 experiments was performed to validate the data on morphological analysis, GABA, calbindin and calretinin immunore- 
activity. Data from early cortical cultures were collected from two ages (E12, $n=2$; E14, $n=1)$ and pooled for quantitative analysis. Likewise, PSVZ experiments ( $\mathrm{P} 2, n=1 ; \mathrm{P} 4, n=2)$ obtained from two ages were pooled together. For BrdU birth dating, the significance was ascertained from four experiments. For quantitative immunocytochemistry, a minimum of 30 fields of view collected from three-four coverslips per experiment were analysed; a field of view $(\times 20$ Nikon objective) on average contained about 200 cells as revealed by DAPI counterstain. For pharmacological assessment, data were obtained from a minimum of four experiments; to measure average $\mathrm{Ca}^{2+}$ activity a total of $30-50$ fields of view were used. Statistical assessment was carried out using unpaired $t$-test with Welch's correction or one-way ANOVA with Bonferroni's multiple comparison tests. Error bars indicate SEM.

\section{Results}

\section{Factors from GE enhance the differentiation of GABAergic} neurons in cortical cultures

It is widely accepted that the phenotypes of cortical interneurons are specified by genetic mechanisms that are specific to the GEs of ventral telencephalon. Thus, we posited that epigenetic factors present in the local environment of GE might enable the differentiation of cortical GABAergic neurons. To test our hypothesis, E16 cortical cells grown in serum-free conditions were exposed to a chronic treatment with conditioned medium collected from E16 GE cultures (GE medium). Examination of treated cortical cultures revealed a significant increase in GABA+ neurons compared with those in serum-free control cultures (Fig. 1A-C; $P<0.001, t$-test). The increase in GABAergic neurons also reflected in the number of $\mathrm{GAD}_{65 / 67}+$ cells in treated cultures (not shown). We next examined whether treatment with GE medium had altered the rate of proliferation or neuronal output in cortical cultures. Analysis of treated cultures pulsed with BrdU for $1 \mathrm{~h}$ showed a similar rate of proliferation in both control and treated cultures (Fig. 1E). Similarly, staining of cultures with MAP2 or Neu$\mathrm{N}$, neuronal markers, did not indicate a significant difference in neuronal output between controls and treated cultures (Fig. 1F). Because the dorsal migration of cortical interneurons is more prevalent during mid-corticogenesis (Nadarajah et al., 2002), we speculated what might be the effect if younger cortical cells were exposed to factors from older GE cultures. Consequently, treatment of E15 cortical cultures with medium collected from age-matched GE cultures (E15) showed an increase in GABA+ neurons; however, the effect was
A

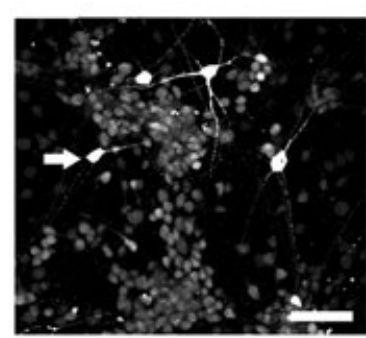

C

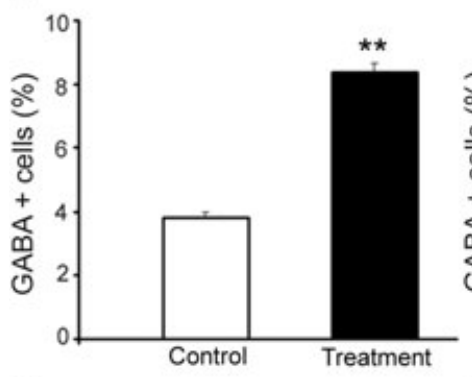

E

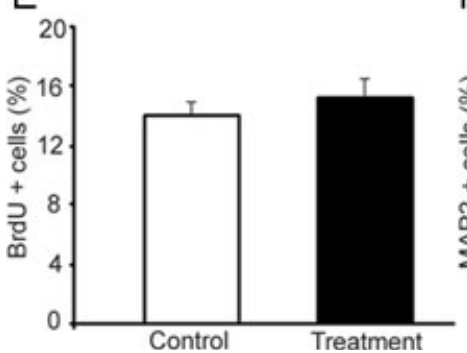

B

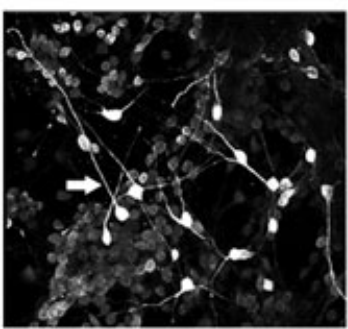

D
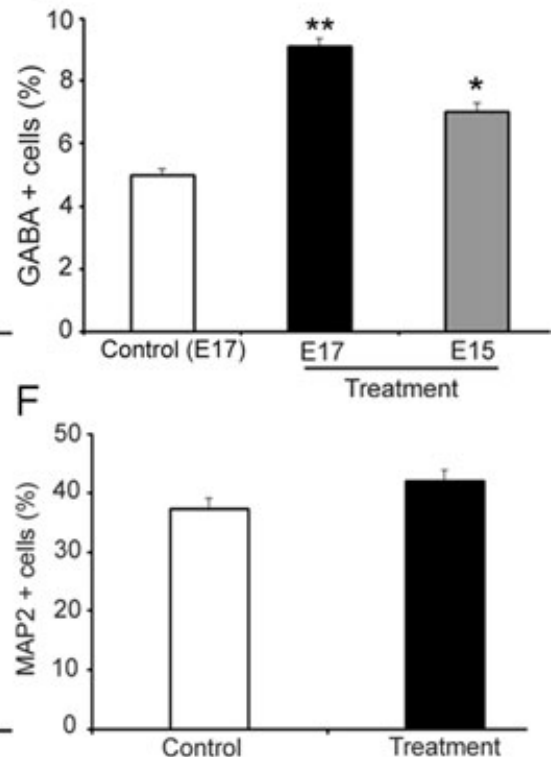

FIG. 1. Characterization of $\gamma$-aminobutyric acid (GABA) immunoreactivity in control and treated cortical cultures. (A-C) GABA-immunoreactive neurons (arrow in A and B) in E16 cortical cultures that were grown in serum-free conditions (A) and in age-matched GE conditioned medium (B). (C) Quantitative analysis in E16 cortical cultures demonstrates a significant difference in the number of GABAergic neurons between control and GE medium $(P<0.001, t$-test). (D) Isochronic and heterochronic experiments using different ages (E17 and E15) of GE medium show that medium from older cultures is more potent in inducing the differentiation of GABAergic neurons. (E and F) Quantitative analysis of E16 control and treated cortical cultures shows a similar rate of cell proliferation (E) and neuronal output (F). Scale bar, $25 \mu \mathrm{m} . * P<0.05$ and $* * P<0.01$. 
A

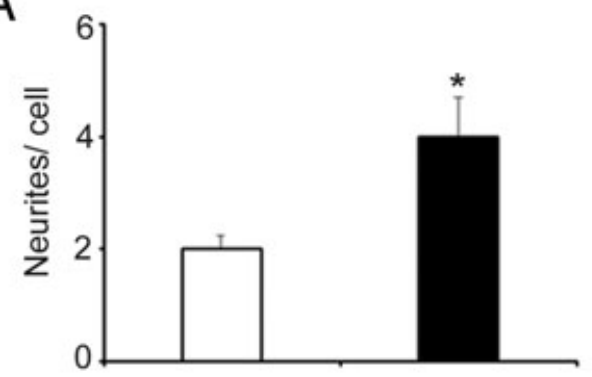

$\mathrm{B}$

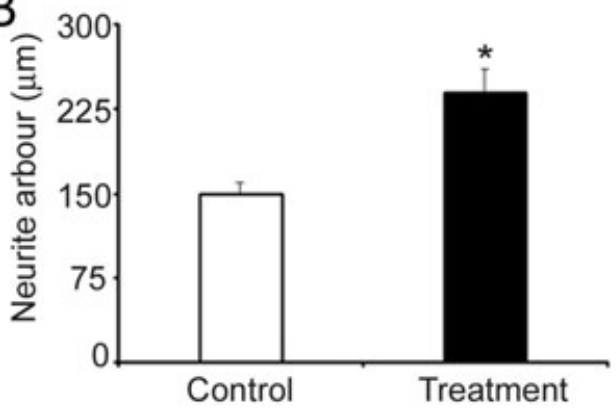

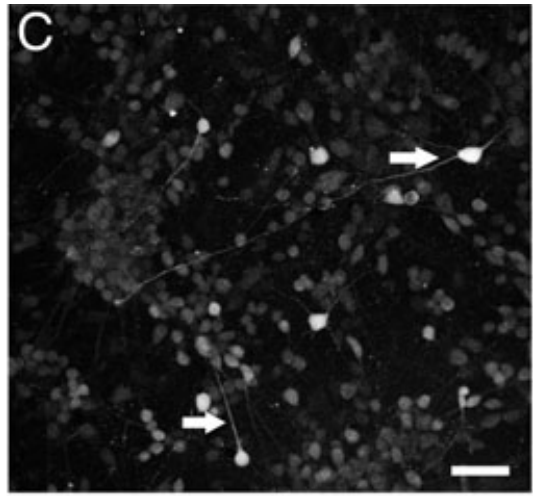

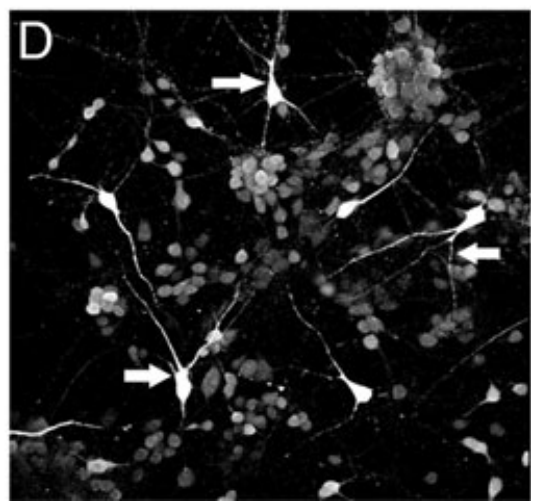

FIG. 2. Morphological characterization of GABAergic neurons in E16 control and treated cultures. Quantitative analysis illustrates significant differences in the number of neurites (A) and in the length of arbour (B) of GABAergic neurons in control and treated cultures $(P<0.01, t$-test). (C and D) Images show GABAergic neurons displaying a varying degree of morphological differentiation (arrows) in control (C) and GE medium (D). Scale bar, $25 \mu \mathrm{m} .{ }^{*} P<0.05$.

more pronounced with medium collected from E17 GE cultures ( $n=3$ experiments, Fig. 1D). Further, careful morphological analysis showed that on average GABA+ neurons in treated cultures were differentiated with more neurites and complex arbor compared with those grown in control medium (Fig. 2A-D). We next investigated whether epigenetic factors from GE could induce the differentiation of GABAergic neurons in other regions of the developing nervous system. For this purpose, we chose to examine the developing spinal cord and mid-brain, as these regions contain GABAergic neurons that are not of ventral telencephalic origin and hence less likely to be influenced by epigenetic factors from GE. Subsequent immunocytochemistry for GABA showed no significant change in E16 mid-brain cultures that were treated with GE medium. However, although application of GE medium to E16 spinal cultures showed a propensity to induce the differentiation of GABAergic neurons, quantitative analysis did not yield a measurable significance (control: $3.1 \pm 0.7 \%$; treatment: $4.72 \pm 0.92 \%, n=3$ experiments).

In vitro studies have shown that cortical progenitors are competent to generate GABA+ cells, contrary to their apparent in vivo behaviour (Gotz et al., 1995; de Lima \& Voigt, 1997; Gulacsi \& Lillien, 2003). Thus, we examined whether chronic exposure to GE medium enables the generation of GABA+ neurons in cortical cultures. Accordingly, serum-free cultures were exposed to BrdU for $1 \mathrm{~h}$ before the application of GE medium to label a cohort of S-phase progenitors to follow their progression over a period of 5 days. Subsequent analysis of both control and treated cultures showed that less than $1 \%$ of GABA+ neurons contained the BrdU label (Fig. 3A and B). Our results illustrate that application of GE medium does not induce the generation of cortical GABAergic neurons any more than the control medium. Nonetheless, GE medium does contain the potency to alter the chemical environment of cortical cells and induce the differentiation of a subset of postmitotic neurons towards GABAergic phenotype without influencing the neuronal output of cortical progenitors.

\section{Factors from GE enhance the differentiation of subtypes of cortical interneurons}

We next examined whether treatment with GE medium could alter the differentiation of subtypes of cortical interneurons. For these experiments, control and treated cultures were stained for calretinin and calbindin to label two of the major subtypes of interneurons in the embryonic cortex. Interestingly, in treated cultures a significant rise was noted in the calretinin+ population compared with controls (Fig. 4E; $n=4$ experiments, $P<0.01, t$-test). Further, these cells were markedly differentiated and appeared to form elaborate networks. In contrast, those in control cultures often appeared as single cells (Fig. 4A and B; arrows). Similarly, calbindin immunoreactivity was also elevated in cortical cultures exposed to GE medium compared with control conditions (Fig. 4F; $n=4$ experiments, $P<0.01, t$-test). Although calbindin is expressed in a subset of projection neurons, the bipolar shape noted in treated cultures was suggestive of interneuronal phenotype (Fig. 4C and D; arrows).

\section{Factors from GE elevate calcium transients in cortical cultures}

Spontaneous calcium $\left(\mathrm{Ca}^{2+}\right)$ transients have been associated with differentiation of spinal neurons (Watt et al., 2000). Moreover, the developmental upregulation of GAD protein in Xenopus spinal 

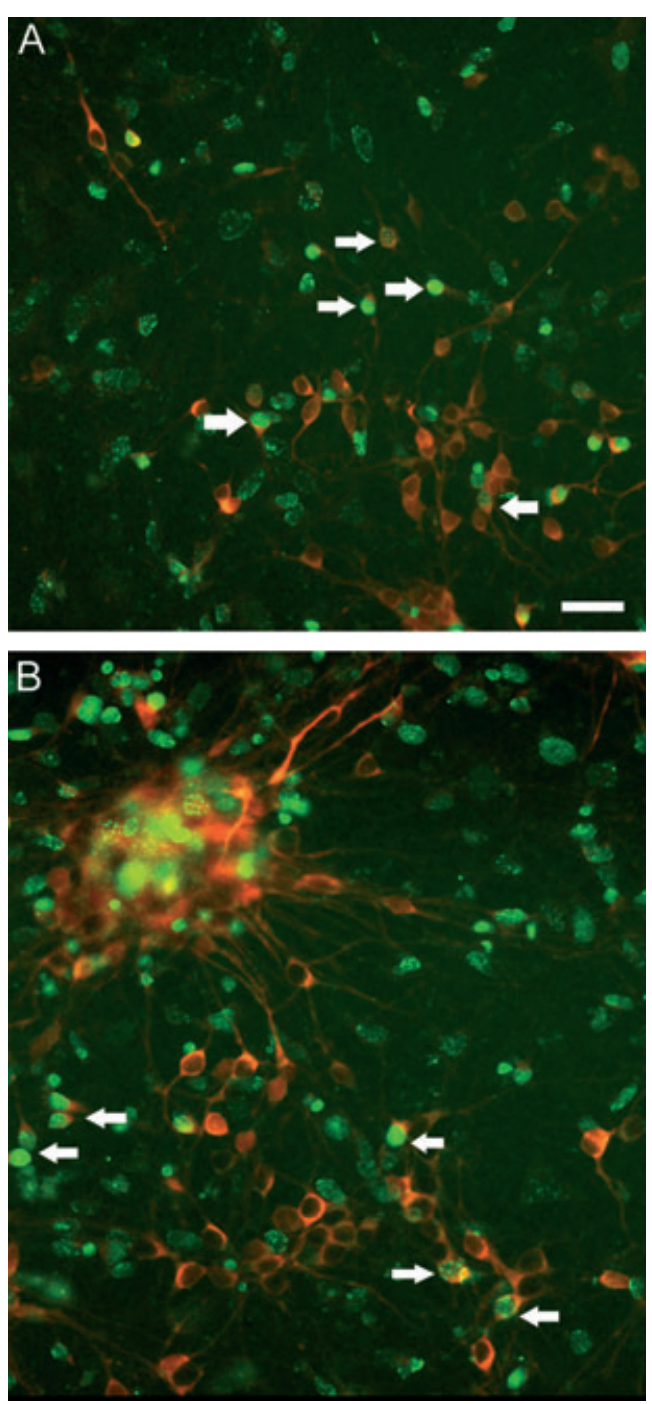

FIG. 3. Generation of GABA+ neurons in E16 control and treated cortical cultures. Birth-dating experiments in control (A) and treated (B) cultures illustrate co-labelling of BrdU (green) with GABA (red); arrows indicate examples of co-labelled cells. No significant difference was noted in the percentage of co-labelling between control and treated cultures. Scale bar, $50 \mu \mathrm{m}$.

neurons has been shown to be $\mathrm{Ca}^{2+}$ dependent (Watt et al., 2000). In the developing cerebral cortex, several patterns of neuronal $\mathrm{Ca}^{2+}$ activity have been described that are mediated by $\mathrm{GABA}_{\mathrm{A}}$ receptor, AMPA and $N$-methyl-D-aspartate (NMDA) glutamate receptors (Owens \& Kriegstein, 2002). While spontaneous transients are due to the release of stored intracellular calcium, synchronized activity between neurons is mediated by synaptic input that is often abolished by TTX, a sodium channel blocker (Spitzer et al., 2004). Gap junctions mediate another form of synchronized transients that occurs primarily between non-neural cells, which is insensitive for TTX treatment (Owens \& Kriegstein, 2002). In our experiments, $\mathrm{Ca}^{2+}$ activity was measured in neural cells as identified by their morphological characteristics. Measurement revealed spontaneously occurring transients in single cells or synchronized activity between cells (Fig. 5A and B). Sampling of many images in treated cultures $(n=50$ fields from over four experiments) showed a marked increase in the number of cells with $\mathrm{Ca}^{2+}$ activity compared with serum-free controls (Fig. 5D; $P<0.001, t$-test). Among the population of cells that displayed spontaneous $\mathrm{Ca}^{2+}$ transients in treated cultures, $40 \pm 2.8 \%$ showed synchronized activity (see Fig. $5 \mathrm{~B}$, cells 1,2 ) in contrast to $15 \pm 2 \%$ of cells in control cultures. Although the duration of $\mathrm{Ca}^{2+}$ transients (average of 2-4 s/transient) did not vary significantly between the two groups, the frequency at which they occurred in single cells was greater in treated cultures (Fig. 5E). Subsequent treatment with acute TTX $(1 \mu \mathrm{m})$ reversibly abolished single transients and synchronized activity in both control and treated cultures; however, random transients in single cells persisted $(5 \pm 1.2 \%$ of active cells; Fig. $5 \mathrm{C})$. Based on their response to TTX, it was clearly evident that the calcium-active cells were indeed of neuronal lineage, and indicates a synaptic input in mediating the synchronized $\mathrm{Ca}^{2+}$ activity in cultures. Our experiments also revealed that the $\mathrm{Ca}^{2+}$ activity was mediated through voltage-dependent calcium channels as acute application of cadmium, a generic blocker of these channels, abolished or reduced the transients in both groups (data not shown).

\section{Induced differentiation of $G A B A+$ neurons: a role for $\mathrm{GABA} /$ glutamate receptors and $\mathrm{Ca}^{2+}$ activity}

How might secreted factors in GE medium influence the $\mathrm{Ca}^{2+}$ activity and increase the GABA immunoreactivity in cortical cultures? GABA is an excitatory neurotransmitter during development and depolarizes cortical cells through the activation of $\mathrm{GABA}_{\mathrm{A}}$ receptors (Owens \& Kriegstein, 2002). Based on the premise that the developing GE contains largely GABAergic neurons, it is likely for the condition medium to contain secreted GABA. Thus, to determine whether bicuculline-sensitive mechanisms mediate the elevated $\mathrm{Ca}^{2+}$ activity, both serum-free cortical cultures and those in GE medium were acutely exposed to $50 \mu \mathrm{m}$ BMI, a selective antagonist of $\mathrm{GABA}_{\mathrm{A}}$ receptor ( $n=4$ experiments, 40 fields). Interestingly, acute application of the antagonist to control cultures abolished the spontaneous transients in $30 \pm 4.5 \%$ of the cells that were initially calcium active (Fig. 6A, cell 1). By contrast, cultures in GE medium were nonresponsive to acute application of the drug as the number of cells that were $\mathrm{Ca}^{2+}$ active or the frequency of transients remained unchanged (Fig. 6B). Next, we examined the pattern of $\mathrm{Ca}^{2+}$ activity in cortical cultures exposed to chronic treatment of BMI alone, or mixed with GE medium. Analysis showed that chronic application of the antagonist in concert with GE medium (BMI + GE) potentiated the $\mathrm{Ca}^{2+}$ transients compared with control cultures (Fig. 6C; $n=4$ experiments, 50 fields, $P<0.01, t$-test). However, the overall activity was significantly less than the measured activity in cells grown in GE medium (compare Fig. 5D with Fig. 6C). To determine whether $\mathrm{GABA}_{\mathrm{A}}$ receptor signalling was involved in the induction of GABA+ neurons, cultures that were exposed to a chronic treatment of BMI were stained for GABA labelling. Immunohistochemical analysis did not reveal a significant difference in the number of GABAergic neurons between cultures maintained in GE medium and those in BMI + GE medium (Fig. 6D, $P>0.05, t$-test). These findings suggest that factors from GE medium are likely to deploy signalling mechanisms other than the $\mathrm{GABA}_{\mathrm{A}}$ receptor pathway to mediate the neurotransmitter differentiation in cortical cultures. Further, exposure of treated cortical cultures to $\mathrm{GABA}_{\mathrm{B}}$ receptor antagonist (50 $\mu \mathrm{m}$ CGP55845A) or to nipecotic acid $(50 \mu \mathrm{m})$, GABA uptake blocker, did not show a significant difference in GABA immunoreactivity compared with control conditions (data not shown).

To explore the involvement of glutamate receptors in mediating the $\mathrm{Ca}^{2+}$ activity and increase in GABA + neurons, serum-free cortical cultures and those in GE medium were acutely exposed to a mixture of $50 \mu \mathrm{m}$ AP5 (NMDA receptor antagonist) and $30 \mu \mathrm{m}$ NBQX 

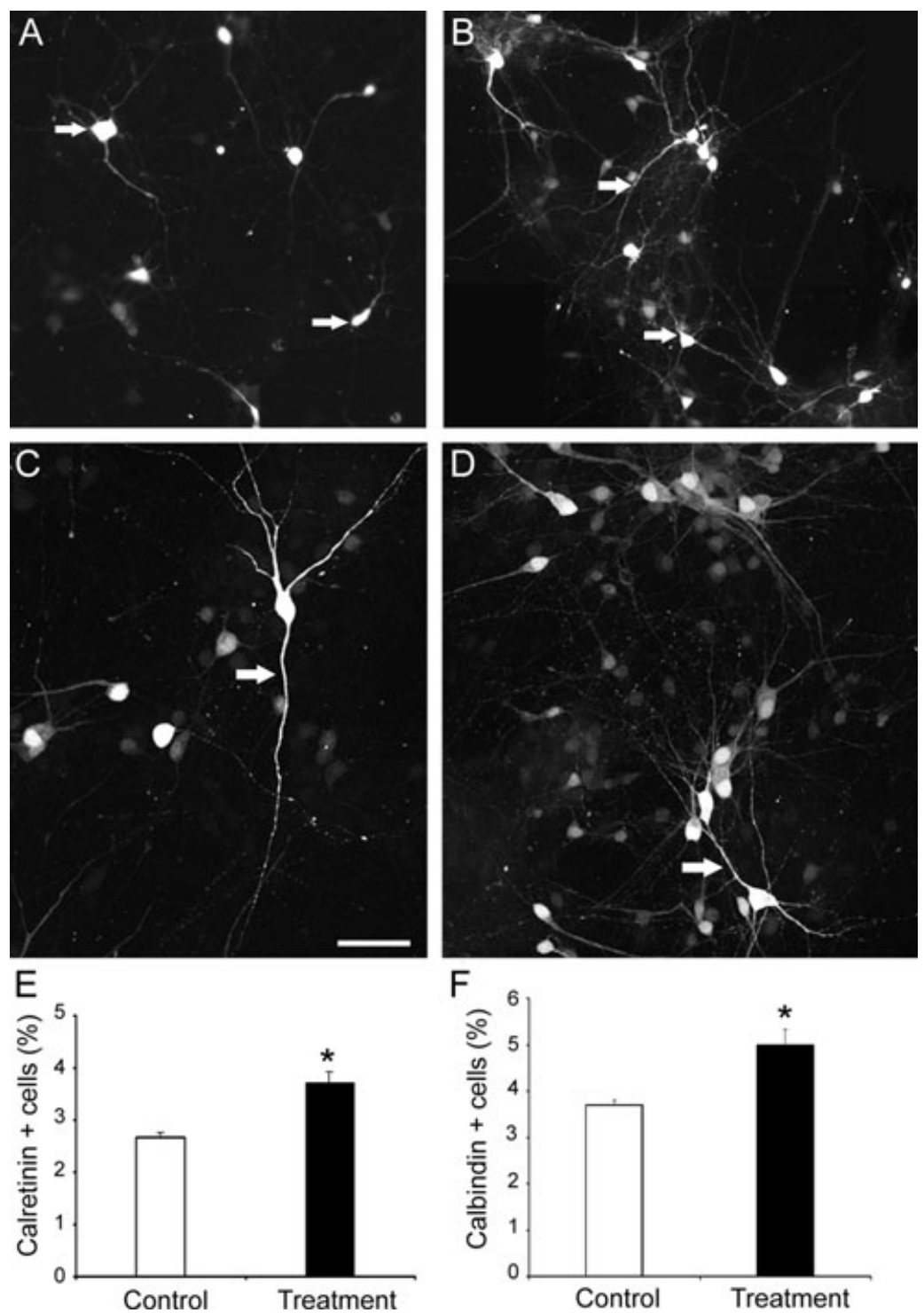

FIG. 4. Characterization of E16 control and treated cortical cultures for subtypes of interneurons. (A and B) Images of calretinin-labelled neurons (arrows) in control (A) and GE medium (B). (C and D) Images of calbindin-labelled neurons (arrows) in control (C) and GE medium (D). (E and F) Illustrating the change in calretinin- (E) and calbindin- (F) labelled neurons following treatment with GE medium. Scale bar, $25 \mu \mathrm{m} .{ }^{*} P<0.05$.

(AMPA/kainate receptor antagonist). Interestingly, an immediate response to the blockers was detected; $\mathrm{Ca}^{2+}$ activity in control cultures reduced to $46 \pm 2 \%$ of the initially active population (Fig. 7A and $\mathrm{C}$ ). By contrast, a significant reduction was observed in GEtreated cultures, as only $28 \pm 1.3 \%$ of the initially active cells continued to display spontaneous $\mathrm{Ca}^{2+}$ transients (Fig. 7B and $\mathrm{C}$, $n=4$ experiments, 40 fields, $P<0.001, t$-test). Staining of cultures grown in chronic AP5 + NBQX alone or together with GE medium $(\mathrm{AP5}+\mathrm{NBQX}+\mathrm{GE})$ also showed a decrease in GABA immunoreactivity compared with cultures grown in GE medium (Fig. 7D, $P<0.001, n=4$ experiments, one-way ANOVA). Next, to determine the effect of individual antagonists, serum-free cortical cultures were subjected to a chronic treatment with AP5, NBQX alone or in concert with GE medium (AP5 + GE or NBQX + GE), respectively. Imaging experiments demonstrated reduced $\mathrm{Ca}^{2+}$ activity in AP5 $+\mathrm{GE}$ and $\mathrm{NBQX}+\mathrm{GE}$ test groups, compared with the average activity measured in cultures grown in GE medium (Fig. 7E and G, $n=4$ experiments, 40 fields, $P<0.001$, one-way ANOVA). Consistent with the $\mathrm{Ca}^{2+}$ data, GABA immunoreactivity was also substantially reduced in AP5 + GE and NBQX + GE cultures, compared with the earlier findings noted in cultures treated with GE medium (Fig. 7F and $\mathrm{H}$, $P<0.001$; one-way ANOVA, $n=4$ experiments).

Recent reports have highlighted a role for brain-derived neurotrophic factor (BDNF) in the potentiated release of glutamate in postnatal cortical neurons (Numakawa et al., 2002). Thus, to determine whether BDNF, a candidate factor in the GE medium, potentiates the release of glutamate and thereby leads to the activation of glutamate receptors, cortical cultures were acutely incubated with K252a or together with GE medium. K252a, a tyrosine receptor kinase B (TrkB) inhibitor, has been shown to prevent the BDNF-evoked release of glutamate through the TrkB signalling pathway. Imaging of serum-free cortical cultures incubated acutely with K252a showed a decrease in $\mathrm{Ca}^{2+}$ activity, as only $35 \pm 4.2 \%$ of the initially active cells continued to display spontaneous transients (Fig. 8A, $n=3$ experiments, 30 fields). By contrast, cells in GE medium were nonresponsive to acute application of K252a (Fig. 8B). Further, chronic 
A
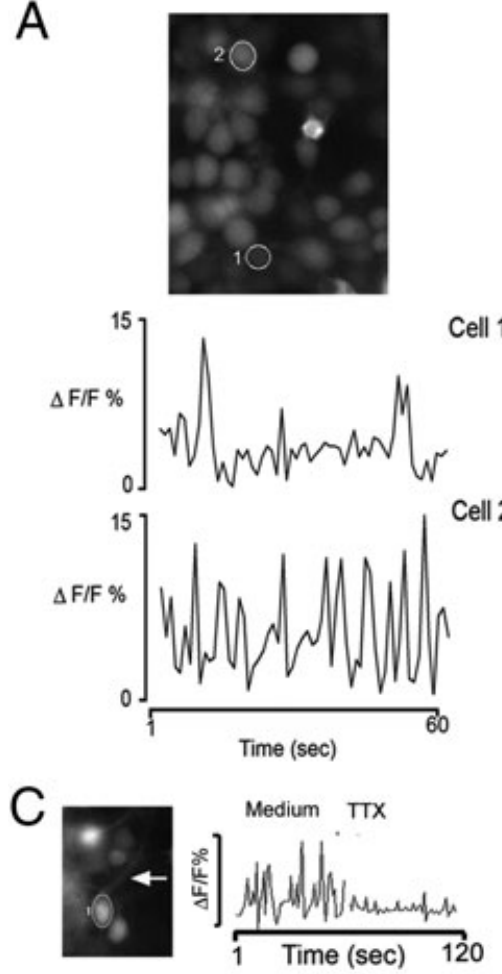

D

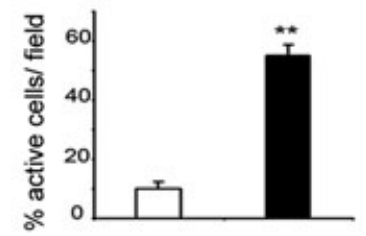

E

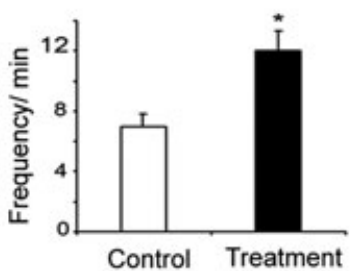

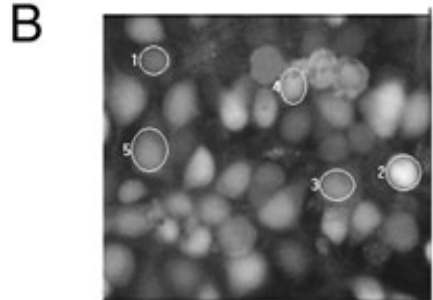

$\Delta F / F \%$

Cell 1

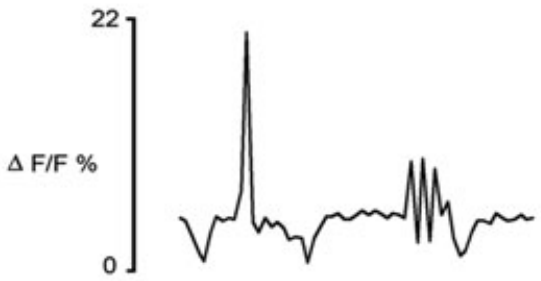

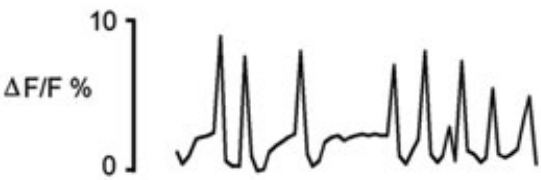

Cell 3

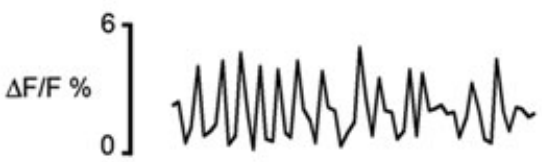

Cell 4

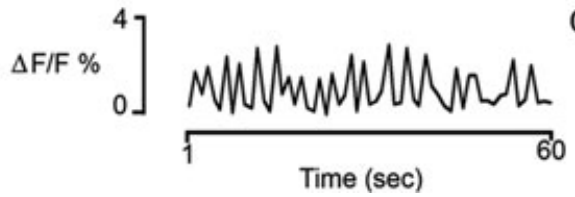

Cell 2

FIG. 5. Measurement of calcium activity in E16 control and treated cortical cultures. (A and B) Measurement of Ca ${ }^{2+}$ activity in control (A) and GE medium (B) cultures illustrates spontaneous transients in single cells or synchronized activity between cells (B; cells 1, 2). (C) Acute application of tetrodotoxin (TTX) abolished $\mathrm{Ca}^{2+}$ transients in control and treated cultures; the illustration is taken from a control culture. (D and E) Quantitative analysis of control and treated cultures illustrates a significant difference in the percentage of cells that showed $\mathrm{Ca}^{2+}$ activity $(P<0.001, t$-test $)$ (D) and in the frequency of transients in single cells (E) $\left(P<0.01, t\right.$-test). ${ }^{*} P<0.05$ and ${ }^{* *} P<0.01$.

treatment of cortical cultures with K252a alone or together with GE medium did not elicit a significant change in $\mathrm{Ca}^{2+}$ activity or in GABA immunoreactivity (data not shown). These results suggest that the activation of glutamate receptors in cells exposed to GE medium is likely to occur through mechanisms that are independent of the $\mathrm{BDNF} / \mathrm{TrkB}$ signalling pathway.

\section{Potency of factors from GE medium with progressing cortical ontogeny}

To determine whether the inducing property of GE medium is intrinsic to the constituent factors, or dependent on the host (cortical) environment, we chose two developmental stages that are well apart in the time line of ontogeny. Thus, early cortical cultures comprising mitotically active multipotent precursors were prepared from E12-14 brains. In contrast to the early stage, PSVZ cultures were obtained from P2-4 brains. In this context, it is pertinent to note that PSVZ progenitors are lineage restricted and hence have reduced potency to generate cells of multiple phenotypes, compared with embryonic progenitors (Vaccarino et al., 2001). In both sets of experiments, cultures were treated with E16 GE medium and analysed for BrdU and GABA immunoreactivity. Analysis of E12-14 cortical cultures showed an increase in the co-labelling of BrdU $+/ \mathrm{GABA}+$ cells compared with age-matched controls. Accordingly, in control cultures $5.64 \pm 1.4 \%$ of BrdU-labelled cells were co-labelled with GABA compared with a marked increase of $26.04 \pm 3.8 \%$ in the treated group ( $n=3$ experiments, $P<0.001, t$-test, for BrdU/GABA staining pattern see Fig. 3). By contrast, application of GE medium to PSVZ 

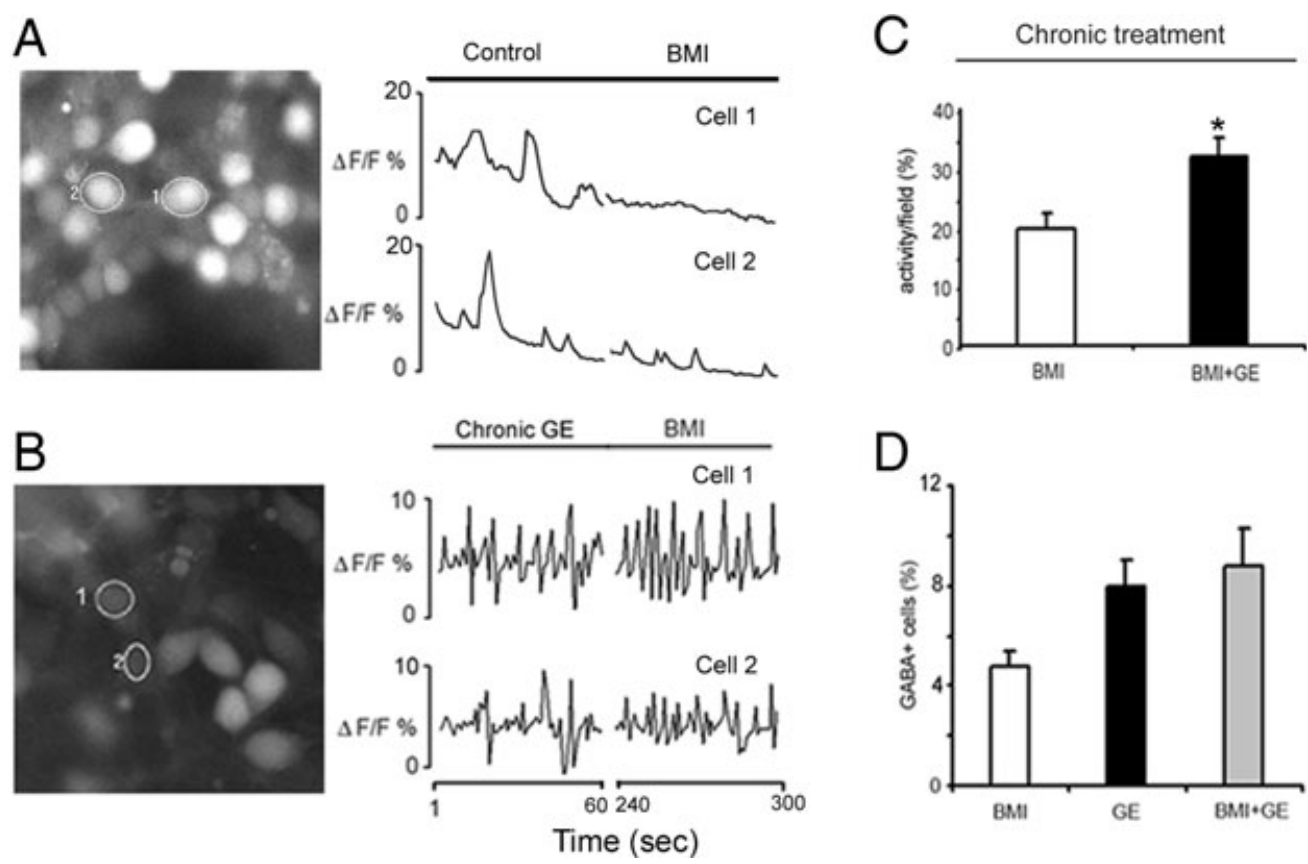

FIG. 6. Application of $\gamma$-aminobutyric acid $(\mathrm{GABA})_{\mathrm{A}}$ receptor antagonist to E16 control and treated cortical cultures. (A and B) Acute application of bicuculline methiodide (BMI) to control (A) cultures abolished spontaneous transients in a third of calcium-active cells (cell 1), while treated cortical cultures (B) were nonresponsive to the drug. (C) Chronic treatment of BMI + ganglionic eminence (GE) medium showed potentiated $\mathrm{Ca}^{2+}$ activity compared with controls (BMI). However, the overall activity in BMI + GE medium cultures was less compared with the measured activity in GE medium (compare Fig. 5D). (D) Quantitative analysis shows that chronic application of BMI does not diminish the differentiation of GABAergic neurons in BMI + GE cultures. $* P<0.05$.

cultures diminished the GABAergic population; in control cultures $52 \pm 2.2 \%$ of neurons were GABA + in comparison to $33 \pm 0.85 \%$ in treated cultures $(n=3$ experiments, $P<0.001, t$-test $) . \quad$ Further characterization of cultures showed that GE treatment does indeed reduce proliferation in PSVZ cells as illustrated by BrdU labelling; $69 \pm 3.1 \%$ BrdU content in controls compared with $46 \pm 5.2 \%$ in treated cultures $(n=3$ experiments, $P<0.01, t$-test). Collectively, these results illustrate that the potency of GE medium to induce the differentiation of GABAergic neurons is largely dependent on the stage of maturation of cortical progenitors, as early progenitors are multipotent and amenable to extrinsic manipulations compared with their lineage-restricted counterparts in mid corticogenesis and early postnatal life.

\section{Discussion}

Using primary cortical cultures, we have examined whether the neurochemical differentiation of cortical interneurons is strictly governed by genetic mechanisms, as widely believed, or is amenable to extrinsic factors. Our experiments have demonstrated that secreted factors from ventral telencephalon have the potency to induce the differentiation of a population of cortical neurons towards GABAergic lineage with an increase in calbindin and calretinin subtypes. Concurrent to neurochemical changes, cortical neurons also showed elevated calcium transients after chronic exposure to GE medium, mediated through the activation of ionotropic glutamate receptors.

\section{Epigenetic influence on neurotransmitter specification of cortical interneurons}

Using BrdU birth-dating experiments, the present study has demonstrated that cortical progenitors grown in serum-free control conditions have the ability to generate GABA+ neurons. Further, chronic exposure of cortical cultures to medium derived from ventral telencephalon induced the differentiation of GABAergic neurons without altering cell proliferation or neuronal output. Increasing evidence now points towards a distinct origin and lineage for the cortical projection and interneuron populations, suggesting an interplay of genetic mechanisms that underlie the specification and differentiation of these cells (Schuurmans \& Guillemot, 2002). Among the early ventral telencephalic genes Dlxl, 2 and Mash1 have been identified to direct the timing and specification of cortical interneurons (Anderson et al., 1997; Fode et al., 2000). How might we reconcile the data from our experiments with the current thesis on the origin of cortical interneurons? Several complementary mechanisms may account for our findings. First, it is possible for the existence of a subset of postmitotic cortical neurons that are yet to assume a neurotransmitter phenotype. In this context, it is noteworthy that the previous cell culture work of Gotz \& Bolz (1994) has shown that initially $\sim 40 \%$ of cortical neurons, although postmitotic, remain undifferentiated with regard to their neurotransmitter type. However, when maintained for prolonged periods the neurotransmitter expression continued to increase with proportions corresponding to in vivo composition. Given the lack of inductive signals such as sonic protein and the presence of BMP, an inhibitory molecule in the dorsal telencephalon, the cortical environment does not support the production of interneurons in vivo (Gulacsi \& Lillien, 2003). Consistent with this notion, it might be posited that upon dissociation of the intact cortex, the inhibition that retained a subset of cortical cells in an undifferentiated state is likely to be removed. Thus, it is plausible for epigenetic factors in the GE medium to have influenced the neurotransmitter differentiation of a population of cortical cells towards GABAergic lineage. Nevertheless, can epigenetic influence override the predetermined genetic specifications to enable a switch 

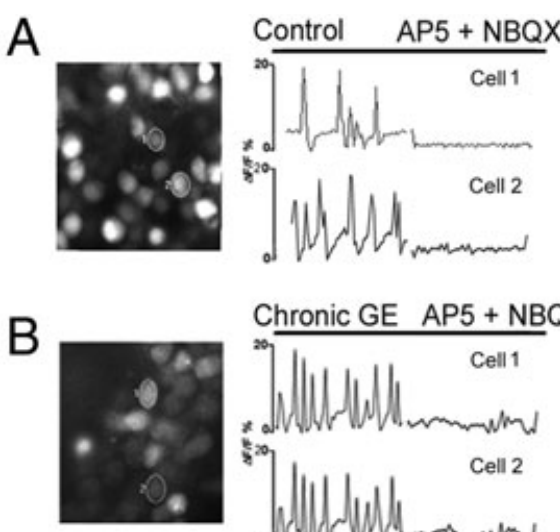
Chronic GE AP5 + NBQX
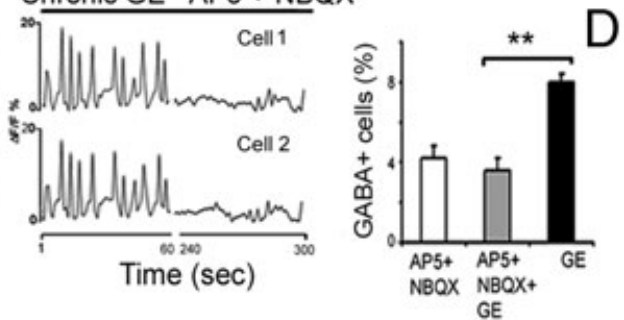

E

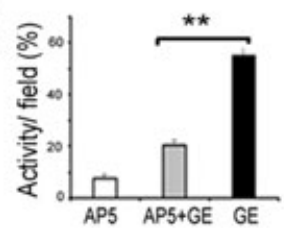

$\mathrm{F}$

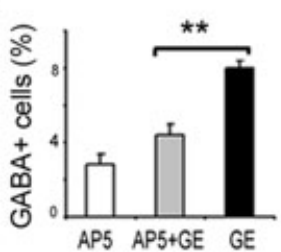

FIG. 7. Application of NMDA and AMPA/kainate receptor antagonists to E16 control and treated cortical cultures. (A-C) Acute application of 2-amino5-phosphonovalerate (AP5) + 1,2,3,4-tetrahydro-6-nitro-2,3-dioxo-benzo[f]quinoxaline-7-sulphonamide (NBQX) to control (A) and treated (B) cultures abolished the spontaneous $\mathrm{Ca}^{2+}$ transients; note the cessation of synchronized activity in (B). (C) Quantitative analysis shows that acute treatment of both antagonists significantly reduced the overall $\mathrm{Ca}^{2+}$ activity in cells maintained in ganglionic eminence (GE) medium compared with controls $(P<0.001, t$-test). (D) Analysis shows that chronic application of AP5 + NBQX together with GE medium (AP5 + NBQX + GE) significantly diminishes the differentiation of GABA+ neurons compared with those grown in GE medium $(P<0.001$, one-way ANOVA). (E and $\mathrm{F})$ Chronic treatment of AP5 together with GE medium (AP5 + GE) reduced $\mathrm{Ca}^{2+}$ activity (E) and GABA immunoreactivity (F), compared with those grown in GE medium $(P<0.001$, one-way ANOvA). (G and $\mathrm{H}$ ) Chronic treatment of NBQX together with GE medium (NBQX $+\mathrm{GE})$ reduced $\mathrm{Ca}^{2+}$ activity (G) and GABAergic neurons $(\mathrm{H})$, compared with those grown in GE medium $\left(P<0.001\right.$, one-way ANOVA). ${ }^{* *} P<0.01$.

from dorsal to ventral neural phenotypes? Interestingly, in a number of mutants with loss of function, cells that transgressed the cortex from GE maintained their specification as ventral cells and continued to express Lhx6, Dlx1/5 and GAD67 (Anderson et al., 1997, 2001; Pleasure et al., 2000). In contrast, transgenic mice with the lacZ gene in the neurogenin 2 (Ngn2) locus have shown that ectopic cortical cells that entered the GE appeared to have changed their fate by downregulating the cortex-specific genes and acquired $D l \times 5$, characteristic of ventral telencephalon (Chapouton et al., 2001). These results, while illustrating the molecular asymmetry that exists between the cell populations of dorsal and ventral telencephalon, further highlight that cortical cells are amenable to environmental influence as they can switch their genetic identity to assume alternative fates. Thus, in a hierarchical system of cell fate specification in the developing cortex, it is possible for epigenetic factor in part to influence the phenotype of cortical interneurons. In support of this view, a recent study has reported that in short-term cultures of hippocampal granule cells, which constitutively express glutamate, GABA and GAD67 can be re-induced by experimental manipulation (Gómez-Lira et al., 2005).

A second possibility for the increase in GABAergic neurons in our test group may have risen from the $D l x+$ cells that apparently migrate from the lateral ganglionic eminence to the cortical proliferative zone during mid to late neurogenesis (mouse E14.5-16; Anderson et al., 2001). Although these ventral-born cells seed the cortical proliferative zone, they are seemingly postmitotic neurons as revealed by their immunoreactivity to TUJ1. The expression of $D l x 1$ in the cortical proliferative zone in vivo raises the possibility of their differential origin. Incidentally, recent reports have illustrated that subpopulations of cortical progenitors do indeed express $D l x$ that consequently generate GABA+ neurons (Letinic et al., 2002; Bellion et al., 2003). However, our results are in accordance with the work of Anderson et al. (2001), as although exposure to GE medium raised the GABA+ neurons in E16 cortical cultures, cell proliferation and neuronal output remained unchanged. This indicates that treatment with GE medium is likely to have induced the differentiation of a postmitotic population, possibly $D l x+$, that is yet to assume a neurotransmitter phenotype. Further, exposure of embryonic spinal cultures to GE medium did not show a significant rise in GABA population, a feature consistent with the finding that $D l x$ is needed for the expression of GAD65/67 and GABA (Stuhmer et al., 2002).

Detailed characterization of cortical cultures exposed to GE medium illustrated a significant rise in the expression of calretinin and calbindin populations. It is possible that 'proto-GABAergic' neurons differentiated into distinct subtypes depending on the availability of epigenetic factors present in the dorsal telencephalon. Further, the potency of epigenetic factors to generate neurons of ventral lineage appeared to have a temporal correlation with progressing ontogeny. Notably, in early embryonic cortex (E12-14) and in early PSVZ, the generation of GABA+ cells in response to treatment was dependent on proliferation, whereas during midneurogenesis (E16) the upregulation of GABA was independent of cell proliferation. Taken together it is evident that the response of cortical cells to extrinsic factors may have been directed by complementary mechanisms that are dependent on the stage of their lineage restriction.

\section{Calcium activity in neurotransmitter differentiation}

What might be the cellular mechanism that enables $\mathrm{Ca}^{2+}$ activity to induce the differentiation of GABA+ neurons? In a series of studies, Spitzer and colleagues have elegantly shown that the transcription of GAD67, a synthetic enzyme of GABA, can be upregulated by increasing $\mathrm{Ca}^{2+}$ spike activity in embryonic Xenopus spinal neurons (Watt et al., 2000; Spitzer, 2002). Further, experimentally induced changes in the frequency of $\mathrm{Ca}^{2+}$ transients have been correlated with the onset of GABAergic phenotype in neural precursors (Ciccolini et al., 2003; for review, see Spitzer et al., 2004). In light of this recent evidence, it is now posited that neurotransmitter specification is collectively regulated by calcium activity, transcription factors and by signalling proteins. Accordingly, early transcription factors initially specify the preliminary fate of neurons that includes the expression of receptors and ion channels. These, in turn, generate spontaneous spike activity and $\mathrm{Ca}^{2+}$ transients through the modulation of channels and 

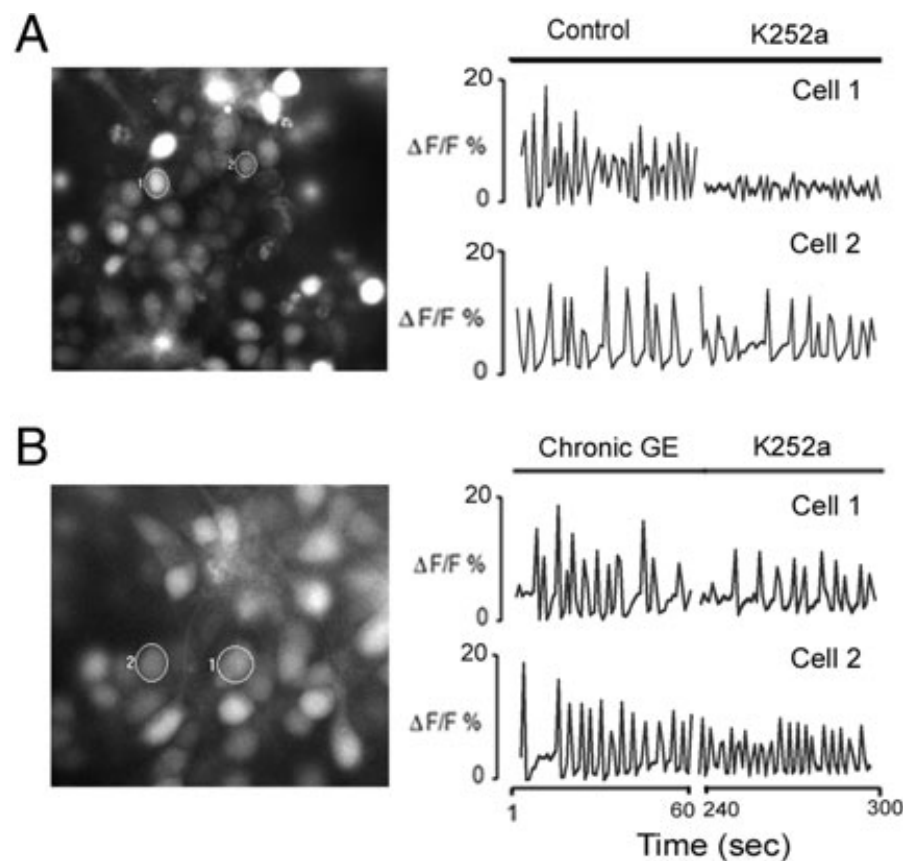

FIG. 8. Application of TrkB receptor antagonist to E16 control and treated cortical cultures. (A) Acute application of K252a to control cultures shows reduced $\mathrm{Ca}^{2+}$ transients in some cells (cell 1), by contrast cells in ganglionic eminence (GE) medium (B) were non-responsive to the drug.

signalling proteins. Patterns of spike activity with the aid of activitydependent transcription factors then regulate the expression of transcripts that encode the synthetic enzymes, transporters and specific transmitters (West et al., 2002; Aizawa et al., 2004; Spitzer et al., 2004).

While a causal relationship is not being suggested, cultures treated with GE medium showed elevated $\mathrm{Ca}^{2+}$ transients that appeared to correlate with a rise in GABAergic neurons. The robust and synchronized $\mathrm{Ca}^{2+}$ activity of the treatment group was responsive to acute TTX and cadmium. Although acute application of BMI to cultures in GE medium did not elicit a significant response, chronic treatment of the blocker reduced the overall $\mathrm{Ca}^{2+}$ activity in $\mathrm{BMI}+\mathrm{GE}$ cultures compared with the activity of cells in GE medium. This indicates that some of the $\mathrm{Ca}^{2+}$ transients noted in cells grown in GE medium were mediated by postsynaptic $\mathrm{GABA}_{\mathrm{A}}$ receptors. Nonetheless, chronic application of BMI together with GE medium was ineffective in reducing the number of GABAergic neurons, suggesting that $\mathrm{GABA}_{\mathrm{A}}$ receptor signalling may not be involved in the upregulation of this inhibitory neurotransmitter. A recent study has demonstrated that tangentially migrating cortical neurons in the intermediate zone, presumably GABA+ neurons, contain NMDA and non-NMDA receptors (Soria \& Valdeolmillos, 2002). Several studies have also reported that ionotropic glutamate receptors are expressed in early postmitotic neurons (Luján et al., 2005). In light of these findings, exposure to acute AP5 + NBQX abolished the synchronized $\mathrm{Ca}^{2+}$ activity in control and GE mediumtreated cultures, indicating the presence of functional glutamate receptors. Further, chronic application of both blockers together with GE medium significantly reduced the $\mathrm{Ca}^{2+}$ activity and the GABAergic neurons, suggesting that ionotropic glutamate receptors were the key elements between $\mathrm{Ca}^{2+}$ transients and neurotransmitter phenotype. Previous studies have reported that BDNF induces glutamate release and potentiates the synchronized $\mathrm{Ca}^{2+}$ oscillations in cultured cortical neurons (Numakawa et al., 2002). In a separate study, Gómez-Lira et al. (2005) have shown that in cultures of hippocampal granule cells GABAergic markers could be re-induced by direct activation of glutamate and TrkB receptors. Thus, to determine whether BDNF, a candidate factor in the GE medium, is involved in potentiating the $\mathrm{Ca}^{2+}$ activity and thereby in the differentiation of GABAergic neurons, control and GE mediumtreated cultures were exposed to K252a. However, blockade of TrkB receptor, either acutely or in chronic treatment, was ineffective in reducing the $\mathrm{Ca}^{2+}$ activity or GABA + neurons, suggesting that BDNF may not be playing an active role in the release of glutamate or potentiating the synchronized $\mathrm{Ca}^{2+}$ activity.

\section{Exposure of cortical neurons to ventral forebrain factors: in vivo significance}

Recent studies have identified GABA and glutamatergic cells in the developing cerebral wall and have shown their differential effects on cortical progenitors (Lidow \& Rakic, 1995; Haydar et al., 2000). Based on the expression data, it has been suggested that decreasing levels of GABA and glutamate in the cortical ventricular zone (VZ) have a propensity to lengthen the cell cycle with progressing neurogenesis (Haydar et al., 2000). Furthermore, postmitotic neurons express both AMPA/kainate and NMDA receptors (Lujan et al., 2005), implying that activation of these glutamate receptors is likely to induce their differentiation and initiate migration. Because GE-born neurons are known to populate the cortical proliferative zone through tangential and ventricle-directed migration (Anderson et al., 2001; Nadarajah et al., 2002), it is conceivable for these neurons to be the source of secreted factors from ventral telencephalon. In this context, at least GABA can be released in a paracrine manner to activate its own receptors (Demarque et al., 2002). Thus, epigenetic factors from ventral telencephalon can be locally released to activate the glutamate receptors of cortical cells, which together with spontaneous $\mathrm{Ca}^{2+}$ activity in the VZ (Owens \& Kriegstein, 1998) is likely to induce the neurotransmitter differentiation of a subset of cortical neurons towards GABAergic phenotype. 


\section{Acknowledgements}

The authors thank Gemma Hughes for her contribution, and Prof. Alex Verkhratsky for his intellectual input in the calcium analysis. This work was supported by grants from the Wellcome Trust (B.N., 070090; J.G.P., 064827).

\section{Abbreviations}

AP5, 2-amino-5-phosphonovalerate; BDNF, brain-derived neurotrophic factor; BMI, bicuculline methiodide; BrdU, bromodeoxyuridine; E, embryonic; GABA, $\gamma$-aminobutyricacid; GE, ganglionic eminence; NBQX, 1,2,3,4-tetrahydro-6nitro-2,3-dioxo-benzo[f]quinoxaline-7-sulphonamide; NMDA, $N$-methyl-Daspartate; P, postnatal; PSVZ, postnatal subventricular zone; TrkB, tyrosine receptor kinase B; TTX, tetrodotoxin; VZ, ventricular zone.

\section{References}

Aizawa, H., Hu, S.C., Bobb, K., Balakrishnan, K., Ince, G., Gurevich, I., Cowan, M. \& Ghosh, A. (2004) Dendrite development regulated by CREST, a calcium-regulated transcriptional activator. Science, 303, 197-202.

Anderson, S.A., Eisenstat, D.D., Shi, L. \& Rubenstein, J.L. (1997) Interneuron migration from basal forebrain to neocortex: dependence on Dlx genes. Science, 17, 474-476.

Anderson, S.A., Marin, O., Horn, C., Jennings, K. \& Rubenstein, J.L. (2001) Distinct cortical migrations from the medial and lateral ganglionic eminences. Development, 128, 353-363.

Bellion, A., Wassef, M. \& Metin, C. (2003) Early differences in axonal outgrowth, cell migration and GABAergic differentiation properties between the dorsal and lateral cortex. Cereb. Cortex, 13, 203-214.

Chapouton, P., Schuurmans, C., Guillemot, F. \& Gotz, M. (2001) The transcription factor neurogenin 2 restricts cell migration from the cortex to the striatum. Development, 128, 5149-5159.

Ciccolini, F., Collins, T.J., Sudhoelter, J., Lipp, P., Berridge, M.J. \& Bootman, M.D. (2003) Local and global spontaneous calcium events regulate neurite outgrowth and onset of GABAergic phenotype during neural precursor differentiation. $J$. Neurosci., 23, 103-111.

Dayer, A.G., Cleaver, K.M., Abouantoun, T. \& Cameron, H.A. (2005) New GABAergic interneurons in the adult neocortex and striatum are generated from different precursors. J. Cell Biol., 168, 415-427.

DeFelipe, J. (1997) Types of neurons, synaptic connections and chemical characteristics of cells immunoreactive for calbindin-D28K, parvalbumin and calretinin in the neocortex. J. Chem. Neuroanat., 4, 1-19.

Demarque, M., Represa, A., Becq, H., Khalilov, I., Ben-Ari, Y. \& Aniksztejn, L. (2002) Paracrine intercellular communication by a Ca2+- and SNAREindependent release of GABA and glutamate prior to synapse formation. Neuron, 36, 1051-1061.

Flames, N. \& Marin, O. (2005) Developmental mechanisms underlying the generation of cortical interneuron diversity. Neuron, 5, 377-381.

Fode, C., Ma, Q., Casarosa, S., Ang, S.L., Anderson, D.J. \& Guillemot, F. (2000) A role for neural determination genes in specifying the dorsoventral identity of telencephalic neurons. Genes Dev., 14, 67-80.

Gómez-Lira, G., Lamas, M., Romo-Parra, H. \& Gutierrez, R. (2005) Programmed and induced phenotype of the hippocampal granule cells. J. Neurosci., 25, 6939-6946.

Gotz, M. \& Bolz, J. (1994) Differentiation of transmitter phenotypes in rat cerebral cortex. Eur. J. Neurosci., 6, 18-32.

Gotz, M., Williams, B.P., Bolz, J. \& Price, J. (1995) The specification of neuronal fate: a common precursor for neurotransmitter subtypes in the rat cerebral cortex in vitro. Eur. J. Neurosci., 7, 889-898.
Gulacsi, A. \& Lillien, L. (2003) Sonic hedgehog and bone morphogenetic protein regulate interneuron development from dorsal telencephalic progenitors in vitro. J. Neurosci., 23, 9862-9872.

Haydar, T.F., Wang, F., Schwartz, M.L. \& Rakic, P. (2000) Differential modulation of proliferation in the neocortical ventricular and subventricular zones. J. Neurosci., 20, 5764-5774.

Kawaguchi, Y. \& Kondo, S. (2002) Parvalbumin, somatostatin and cholecystokinin as chemical markers for specific GABAergic interneuron types in the rat frontal cortex. J. Neurocytol., 31, 277-287.

Letinic, K., Zoncu, R. \& Rakic, P. (2002) Origin of GABAergic neurons in the human neocortex. Nature, 417, 645-649.

Lidow, M.S. \& Rakic, P. (1995) Neurotransmitter receptors in the proliferative zones of the developing primate occipital lobe. J. Comp. Neurol., 360, 393402.

de Lima, A.D. \& Voigt, T. (1997) Identification of two distinct populations of gamma-aminobutyric acidergic neurons in cultures of the rat cerebral cortex. J. Comp. Neurol., 388, 526-540.

Luján, R., Shigemoto, R. \& López-Bendito, G. (2005) Glutamate and GABA receptor signalling in the developing brain. Neuroscience, 130, 567-580.

Marin, O. \& Rubenstein, J.L. (2001) A long, remarkable journey: tangential migration in the telencephalon. Nat. Rev. Neurosci., 2, 780-790.

Markram, H., Toledo-Rodriguez, M., Wang, Y., Gupta, A., Silberberg, G. \& $\mathrm{Wu}, \mathrm{C}$. (2004) Interneurons of the neocortical inhibitory system. Nat. Rev. Neurosci., 5, 793-807.

Nadarajah, B., Alifragis, P., Wong, R.O. \& Parnavelas, J.G. (2002) Ventricledirected migration in the developing cerebral cortex. Nat. Neurosci., 5, $218-$ 224.

Numakawa, T., Yamagishi, S., Adachi, N., Matsumoto, T., Yokomaku, D., Yamada, M. \& Hatanaka, H. (2002) Brain-derived neurotrophic factorinduced potentiation of $\mathrm{Ca}^{2+}$ oscillations in developing cortical neurons. J. Biol. Chem., 277, 6520-6529.

Owens, D.F. \& Kriegstein, A.R. (1998) Patterns of intracellular calcium fluctuation in precursor cells of the neocortical ventricular zone. J. Neurosci., 18, 5374-5388.

Owens, D.F. \& Kriegstein, A.R. (2002) Is there more to GABA than synaptic inhibition? Nat. Rev. Neurosci., 3, 715-727.

Pleasure, S.J., Anderson, S., Hevne, R., Bagri, A., Marin, O., Lowenstein, D.H., Rubenstein, J. \& L. (2000) Cell migration from the ganglionic eminences is required for the development of hippocampal GABAergic interneurons. Neuron, 28, 727-740.

Schuurmans, C. \& Guillemot, F. (2002) Molecular mechanisms underlying cell fate specification in the developing telencephalon. Curr. Opin. Neurobiol., 12, 26-34.

Soria, J.M. \& Valdeolmillos, M. (2002) Receptor-activated calcium signals in tangentially migrating cortical cells. Cereb. Cortex, 12, 831-839.

Spitzer, N.C. (2002) Activity-dependent neuronal differentiation prior to synapse formation: the functions of calcium transients. J. Physiol. Paris, 96, 73-80.

Spitzer, N.C., Root, C.M. \& Borodinsky, L.N. (2004) Orchestrating neuronal differentiation: patterns of $\mathrm{Ca} 2+$ spikes specify transmitter choice. Trends Neurosci., 27, 415-421.

Stuhmer, T., Puelles, L., Ekker, M. \& Rubenstein, J.L. (2002) Expression from a Dlx gene enhancer marks adult mouse cortical GABAergic neurons. Cereb. Cortex, 12, 75-85.

Vaccarino, F.M., Ganat, Y., Zhang, Y. \& Zheng, W. (2001) Stem cells in neurodevelopment and plasticity. Neuropsychopharmacology, 25, 805-815.

Watt, S.D., Gu, X., Smith, R.D. \& Spitzer, N.C. (2000) Specific frequencies of spontaneous $\mathrm{Ca} 2+$ transients upregulate GAD 67 transcripts in embryonic spinal neurons. Mol. Cell. Neurosci., 16, 376-387.

West, A.E., Griffith, E.C. \& Greenberg, M.E. (2002) Regulation of transcription factors by neuronal activity. Nat. Rev. Neurosci., 3, 921-931. 